\title{
Combined Bulk/Per-Subcarrier Relay Selection in Two-Hop OFDM Systems
}

\author{
Shuping Dang, Justin P. Coon, David E. Simmons \\ Department of Engineering Science, University of Oxford, Oxford, UK, OX1 3PJ \\ Email: \{shuping.dang, justin.coon, david.simmons\}@eng.ox.ac.uk
}

\begin{abstract}
In this paper, we apply the concept of combined bulk/per-subcarrier selection to two-hop relay selection systems employing OFDM. The outage probability of the proposed strategy is analyzed in the high SNR regime when decodeand-forward, fixed-gain amplify-and-forward and variable-gain amplify-and-forward are employed at the relays. Meanwhile, a generalized situation without specifying the relaying protocol is also analyzed. We mathematically prove that the combined selection strategy is able to achieve an optimal outage probability equivalent to conventional per-subcarrier selection in the high SNR regime without using the full set of available relays for selection. Moreover, we demonstrate through numerical simulations that this performance advantage holds when channels are spatially correlated.
\end{abstract}

Index Terms-Relay selection, OFDM, outage probability, combined selection, cooperative network.

\section{INTRODUCTION}

Since the proposal of the cooperative network, the theoretical and implementation issues of relay networks have become important research topics in both industry and academia. As an effective way to exploit spatial and frequency diversity, relay selection has been touted as one of the most promising techniques in the next generation of cooperative wireless networking [1]-[3]. For orthogonal frequency-division multiplexing (OFDM) systems, bulk selection (a single relay is selected for transmission on all subcarriers) and per-subcarrier selection (selection is treated independently for each subcarrier, thus, potentially, leading to transmission via multiple relays) have been proposed [4]. However, the performance of the former is too poor to satisfy the increasingly high requirements of modern networks in terms of reliability and throughput. On the other hand, the latter strategy normally requires too many relays in one transmission interval, and thereby makes the selection and synchronization among the source, the relays and the destination difficult [5].

To solve similar problems in transmit antenna selection (TAS) systems, a novel selection strategy called combined selection was proposed in [6]. In this strategy, only two out of the total available antennas are selected, and per-subcarrier selection is performed over these two selected antennas. Interestingly, OFDM system employing such a suboptimal strategy can achieve the same outage and symbol-error probabilities as systems employing a per-subcarrier selection strategy in the high signal-to-noise ratio (SNR) regime. In this paper, we refer to this asymptotic behavior as the equivalence principle.
It is natural to ask whether the combined selection strategy can be extended to relay selection. Clearly, the answer is "yes" to a certain degree. What is not obvious is whether the equivalence principle holds in relay selection systems, particularly for different forwarding schemes. The main consideration in this case is that the end-to-end SNR statistics exhibit different characteristics than those in TAS systems. In this paper, we mathematically prove that it does indeed hold for cooperative networks employing the three fundamental forwarding schemes: decode-and-forward (DF), fixed-gain (FG) amplifyand-forward (AF), and variable-gain (VG) AF. Meanwhile, a generalized situation without specifying the relaying protocol is also analyzed, and we prove that the equivalence principle is generally valid as long as a general condition can be met. We provide numerical results that validate our analysis, and also demonstrate through simulations that the equivalence principle appears to extend to channels that are spatially correlated.

\section{SySTEM MODEL}

\section{A. Relays and channels}

For a typical OFDM system with a single source and destination, $M$ relays and $K$ subcarriers, if the combined selection strategy first chooses $L=2$ out of $M$ relays, the bulk selection, per-subcarrier selection and combined selection strategies can be illustrated in Fig. 1. It is clear that for $M$ relays and $K$ subcarriers, $2 M K$ channels exist in the spacefrequency grid. For brevity, we tentatively assume that the $2 M K$ channel coefficients are statistically independent, zeromean, complex Gaussian (ZMCG) random variables with total variance $\mu_{i}$, where $i \in\{1,2\}$ corresponds to the first and second hop, respectively. We use $h(m, k)$ to denote the frequencydomain channel coefficients between the source and the $m$ th relay for the $k$ th subcarrier, $\forall m \in \mathcal{M}=\{1,2, \ldots, M\}$, $\forall k \in \mathcal{K}=\{1,2, \ldots, K\}$, and similarly $g(m, k)$ to denote the channel coefficients between the $m$ th relay and the destination for the $k$ th subcarrier. Meanwhile, without loss of generality, if we assume $h(m, k) \sim \mathcal{C N}\left(0, \mu_{1}\right)$ and $g(m, k) \sim \mathcal{C N}\left(0, \mu_{2}\right)$, it is well known that $|h(m, k)|^{2}$ and $|g(m, k)|^{2}$ are distributed as $\Gamma\left(1, \mu_{1}\right)$ and $\Gamma\left(1, \mu_{2}\right)^{1}$.

We further assume that the channel state information (CSI) is perfectly estimated and shared among all communication nodes, and the relay network operates in a half-duplex protocol

\footnotetext{
${ }^{1} \mathcal{C N}(\cdot, \cdot)$ and $\Gamma(\cdot, \cdot)$ represent the Gaussian and the Gamma distributions, respectively.
} 


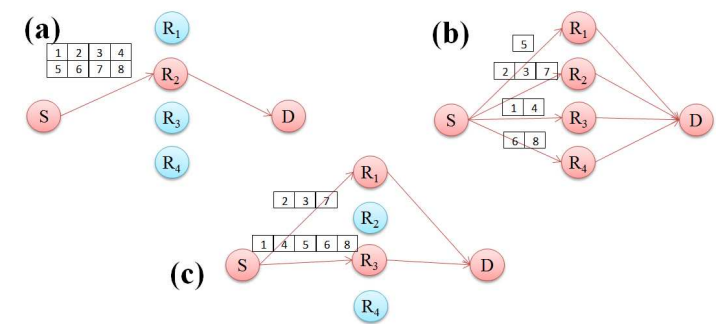

Fig. 1. Illustration of (a) bulk, (b) per-subcarrier and (c) combined bulk/persubcarrier relay selection strategies for single source, single destination andmultiple relays, given $K=8, M=4$ and $L=2$. The numbers in boxes are corresponding to the sequence numbers of subcarriers.

so that two orthogonal time slots are required for one complete transmission from source to destination. The noise statistics on the $k$ th subcarrier at the $m$ th relay and at the destination are denoted by $n(m, k)$ and $\eta(k)$, respectively, and all $M K+K$ statistics are independent, identically distributed (i.i.d.) ZMCG random variables with variance $N_{0} / 2$ per dimension.

Assuming equal bit and power allocation schemes are applied, the average transmit power per subcarrier at the source and at each utilized relay is denoted by $P_{t}$ [7]. Hence, the equivalent instantaneous end-to-end $\mathrm{SNR}^{2}$ corresponding to the $k$ th subcarrier and the $m$ th relay using a DF protocol can be expressed as [8]

$$
\operatorname{SNR}(m, k)=\frac{P_{t}}{N_{0}} \min \left(|h(m, k)|^{2},|g(m, k)|^{2}\right) .
$$

It is also well known that for FG AF relaying, the instantaneous end-to-end SNR can be expressed as [9]

$$
\operatorname{SNR}(m, k)=\frac{G_{F}^{2}|h(m, k)|^{2}|g(m, k)|^{2} P_{t}}{\left(G_{F}^{2}|g(m, k)|^{2}+1\right) N_{0}},
$$

where $G_{F}=\sqrt{P_{t} /\left(P_{t} \mu_{1}+N_{0}\right)}$ is the per-subcarrier relay gain. Finally, for VG AF relaying with per-subcarrier relay gain factor $G_{V}=\sqrt{P_{t} /\left(P_{t}|h(m, k)|^{2}+N_{0}\right)}$, the instantaneous end-to-end SNR can be expressed as [10]

$$
\operatorname{SNR}(m, k)=\frac{|h(m, k)|^{2}|g(m, k)|^{2} P_{t}^{2}}{\left(|g(m, k)|^{2} P_{t}+|h(m, k)|^{2} P_{t}+N_{0}\right) N_{0}} .
$$

\section{B. Relay selection}

Now let us consider the selection process. It has been verified that the outage probability mainly depends on the worst channel over all subcarriers at high SNR and thus we should maximize the minimum channel coefficient over all subcarriers in order to minimize the outage probability [6]. Following this logic, the bulk selection criterion can be expressed as [11]

$$
\hat{\mathcal{L}}_{\text {bulk }}=\arg \max _{\mathcal{L} \subseteq \mathcal{M}} \min _{k \in \mathcal{K}} \max _{l \in \mathcal{L}} \operatorname{SNR}(l, k)
$$

\footnotetext{
${ }^{2}$ In fact, an outage in DF relaying networks depends on the minimum channel coefficient among the source-relay and the relay-destination links. Hence, we can employ the minimum channel coefficient as the equivalent channel quality indicator here.
}

where $\mathcal{L}$ identifies a pair of relays that can be used to carry out per-subcarrier selection. Clearly, $|\mathcal{L}|=2$. Note that we could consider $|\mathcal{L}|>2$, but this is not required to prove our main result, namely that the equivalence principle applies to relay selection systems.

For a given set of relays chosen according to (4), the optimal per-subcarrier selection criterion for subcarrier $k \in \mathcal{K}$ is given by [11]

$$
\hat{l}_{p s}(k)=\arg \max _{l \in \hat{\mathcal{L}}_{\text {bulk }}} \operatorname{SNR}(l, k) .
$$

A note on the practicality of these selection processes is in order. In fact, it is straightforward to see that selection of this nature is practically achievable by adopting a welldesigned timer-based scheme [5]. In this paper, we consider the time interval within which two or more timers expire to be infinitesimal. Hence, we do not need to worry about the timer contention problem here. This is equivalent to assuming that the selection process is perfect, which is justified since we are concerned with theoretical performance limits.

\section{Performance Analysis}

We adopt outage probability as a metric to measure the performance of OFDM systems employing relay selection. In what follows, we define the outage probability in the usual way:

$$
F(s)=\mathbb{P}(\operatorname{SNR}(m, k)<s)
$$

where $s$ is an end-to-end SNR threshold. Our goal is to show that as $P_{t} / N_{0} \rightarrow \infty$, the outage probability for cooperative networks employing combined selection (with $|\mathcal{L}|=2$ ) tends to the outage probability for networks that use per-subcarrier selection over the relay set $\mathcal{L}=\mathcal{M}$. We consider each selection strategy in turn.

\section{A. Combined selection}

Denote the probability density function (PDF) corresponding to $F(s)$ as $f(s)$. Because outage is dominated by the worst channel at high SNR, using Lemma 1 in [6], we obtain the PDF of the outage probability of an OFDM relaying system employing combined bulk/per-subcarrier selection at high SNR [12]:

$$
f_{\text {comb }}(s)=M K[F(s)]^{M-1}[1-F(s)]^{M K-M} f(s) .
$$

The corresponding CDF $F_{\text {comb }}(s)$ of $f_{\text {comb }}(s)$ can be used to quantify the asymptotic outage performance for the selection system.

1) DF relay: Now, let us focus on the DF relay case. Because $|h(m, k)|^{2} \sim \Gamma\left(1, \mu_{1}\right)$ and $|g(m, k)|^{2} \sim \Gamma\left(1, \mu_{2}\right)$, the PDF and CDF of $|h(m, k)|^{2}$ and $|g(m, k)|^{2}, \forall m \in \mathcal{M}$, $\forall k \in \mathcal{K}$ are

$$
f_{w}(s)=e^{-s / \mu_{i}} / \mu_{i} \Leftrightarrow F_{w}(s)=1-e^{-s / \mu_{i}}
$$

for $i \in\{1,2\}$. Define the normalized $S N R$ to be $\bar{\gamma}=P_{t} / N_{0}$. By applying the theory of order statistics, $F(s)$ in DF relaying networks with selection defined according to (1) is given by

$$
F(s)=1-e^{-s\left(1 / \mu_{1}+1 / \mu_{2}\right) / \bar{\gamma}} .
$$


Consequently, the PDF $f(s)$ corresponding to $F(s)$ in DF relaying networks is

$$
f(s)=\frac{\mathrm{d} F(s)}{\mathrm{d} s}=\frac{1}{\bar{\gamma}}\left(\frac{1}{\mu_{1}}+\frac{1}{\mu_{2}}\right) e^{-s\left(\frac{1}{\mu_{1}}+\frac{1}{\mu_{2}}\right) / \bar{\gamma}} .
$$

According to (7), (9) and (10), the PDF of the worst channel at high SNR with the combined bulk/per-subcarrier selection is

$$
\begin{aligned}
f_{\text {comb }}(s) & =\frac{M K}{\bar{\gamma}}\left(\frac{1}{\mu_{1}}+\frac{1}{\mu_{2}}\right) \\
& \times\left[1-e^{-s\left(\frac{1}{\mu_{1}}+\frac{1}{\mu_{2}}\right) / \bar{\gamma}}\right]^{M-1} \\
& \times\left[e^{-s\left(\frac{1}{\mu_{1}}+\frac{1}{\mu_{2}}\right) / \bar{\gamma}}\right]^{M K-M+1} .
\end{aligned}
$$

Meanwhile, the system outage probability $F_{\text {comb }}(s)=$ $\int_{0}^{s} f_{\text {comb }}(t) \mathrm{d} t$ can be expressed by an asymptotic approximation at high SNR $(\bar{\gamma} \rightarrow \infty)$. By taking the leading order term of the Taylor series at $\bar{\gamma} \rightarrow \infty$, we obtain

$$
F_{c o m b}(s) \simeq\left[\frac{K^{\frac{1}{M}} s\left(\mu_{1}+\mu_{2}\right)}{\mu_{1} \mu_{2} \bar{\gamma}}\right]^{M} .
$$

2) $F G$ AF relay: As for the FG AF relay case, using (2), it can be shown that

$$
F(s)=1-2 e^{-\frac{s}{\mu_{1} \bar{\gamma}}} \sqrt{\frac{s\left(\mu_{1}+\frac{1}{\bar{\gamma}}\right)}{\mu_{1} \mu_{2} \bar{\gamma}}} K_{1}\left(2 \sqrt{\frac{s\left(\mu_{1}+\frac{1}{\bar{\gamma}}\right)}{\mu_{1} \mu_{2} \bar{\gamma}}}\right)
$$

where $K_{1}(\cdot)$ is the first order modified Bessel function of the second kind. This is the standard outage result for FG AF networks. Applying (7) and using the series expansion of the Bessel function along with binomial and Taylor expansions of the algebraic and exponential terms in (13) yields the asymptotic outage expression at $\bar{\gamma} \rightarrow \infty$

$$
F_{c o m b}(s) \simeq\left[\frac{K^{\frac{1}{M}} s}{\mu_{2} \bar{\gamma}} \ln (\bar{\gamma})\right]^{M} .
$$

3) VG AF relay: For VG AF relaying, the CDF of (3) has been proved to be [13]

$$
\begin{aligned}
F(s) & =1-2 e^{-\frac{s}{\gamma}\left(\frac{1}{\mu_{1}}+\frac{1}{\mu_{2}}\right)} \sqrt{\frac{s}{\mu_{1} \bar{\gamma}^{2}}\left(\frac{1}{\mu_{2}}+s\right)} \\
& \times K_{1}\left(2 \sqrt{\frac{s}{\mu_{1} \bar{\gamma}^{2}}\left(\frac{1}{\mu_{2}}+s\right)}\right) .
\end{aligned}
$$

Using a similar approach to the FG AF case, we find that

$$
F_{\text {comb }}(s) \simeq\left[\frac{K^{\frac{1}{M}} s\left(\mu_{1}+\mu_{2}\right)}{\mu_{1} \mu_{2} \bar{\gamma}}\right]^{M}
$$

for large $\bar{\gamma}$. Note that the expression given in (16) is exactly the same as that given in (12), which indicates the performance of VG AF relaying is identical to DF relaying at high SNR. A related result was explored in [14]. However, the equivalence of DF and VG AF relay performance in the present context of combined bulk/per-subcarrier selection has not been reported in the literature until now.

\section{B. Per-subcarrier selection}

Considering the PDF of the worst subcarrier when applying per-subcarrier selection, we have that the PDF of the instantaneous end-to-end SNR is given by

$$
f_{p s}(s)=M K[F(s)]^{M-1}\left\{1-[F(s)]^{M}\right\}^{K-1} f(s) .
$$

By repeating the derivations outlined in the previous subsection, the asymptotic expression (leading order term) for the CDF of the system employing per-subcarrier selection, denoted as $F_{p s}(s)$, can be easily calculated for all three kinds of relays. By carrying out these calculations, we find that the asymptotic expressions of $F_{p s}(s)$ with per-subcarrier selection for all three relaying protocols agree with the combined selection results given in (12), (14), and (16). To see this result without going into the details of the specific calculations, one can observe (7) and (17) carefully, noting that both PDFs are dominated by the terms $M K,[F(s)]^{M-1}$ and $f(s)$ for the three relay protocols. Indeed, the differences in the expressions, notably $[1-F(s)]^{M K-M}$ and $\left\{1-[F(s)]^{M}\right\}^{K-1}$, are incorporated into higher order terms in the asymptotic expansion of the outage probability and, hence, do not contribute at high SNR. Note, however, that these corrections will influence the rate of convergence of the combined selection performance to that of per-subcarrier selection. Furthermore, we can propose a generalized theorem and its proof in the next subsection to reveal the mathematical details.

\section{Generalized theorem of equivalence principle}

As we have proved the equivalence principle for three types of relays, one might wonder whether this equivalence principle can be further extended to other applications. To answer this question, a generalized equivalence principle can be stated as follows.

Theorem 1: If the CDF of the i.i.d. end-to-end SNR, $F(s)$, can be expanded in the variable $\bar{\gamma}$ as

$$
F(s)=\sum_{i=i_{0}}^{\infty} c_{i}(s)\left(\frac{1}{\bar{\gamma}}\right)^{\frac{i}{\theta}}[\ln (\bar{\gamma})]^{r}
$$

where $i_{0}$ is an integer given by $i_{0}=\arg \min _{n \in \mathbb{N}}\left\{c_{n}(s) \neq 0\right\}$; $\theta$ is a nonzero natural number; $\left\{c_{i}(s)\right\}$ represents a series of functions of $s ; r \in \mathbb{N}$, then combined selection is able to achieve an outage probability equivalent to conventional persubcarrier selection in the high SNR regime.

Proof: From (18), we can determine $f(s)$ by

$$
f(s)=\frac{\mathrm{d} F(s)}{\mathrm{d} s}=\sum_{i=i_{0}}^{\infty}\left(\frac{1}{\bar{\gamma}}\right)^{\frac{i}{\theta}}[\ln (\bar{\gamma})]^{r} \frac{\mathrm{d} c_{i}(s)}{\mathrm{d} s} .
$$


Therefore, according to (7), the asymptotic expression (leading order term) at $\bar{\gamma} \rightarrow \infty$ for the PDF $f_{\text {comb }}(s)$ can be determined by

$$
\begin{aligned}
& f_{\text {comb }}(s)=M K\left\{\sum_{i=i_{0}}^{\infty} c_{i}(s)\left(\frac{1}{\bar{\gamma}}\right)^{\frac{i}{\theta}}[\ln (\bar{\gamma})]^{r}\right\}^{M-1} \\
& \times\left\{1-\sum_{i=i_{0}}^{\infty} c_{i}(s)\left(\frac{1}{\bar{\gamma}}\right)^{\frac{i}{\theta}}[\ln (\bar{\gamma})]^{r}\right\}^{M K-M} \\
& \times\left\{\sum_{i=i_{0}}^{\infty} \frac{\mathrm{d} c_{i}(s)}{\mathrm{d} s}\left(\frac{1}{\bar{\gamma}}\right)^{\frac{i}{\theta}}[\ln (\bar{\gamma})]^{r}\right\} \\
& \sim M K\left[c_{i_{0}}(s)\right]^{M-1}\left(\frac{1}{\bar{\gamma}}\right)^{\frac{i_{0}}{\theta} M}[\ln (\bar{\gamma})]^{r M} \frac{\mathrm{d} c_{i_{0}}(s)}{\mathrm{d} s} .
\end{aligned}
$$

Note, it is obvious that $c_{i_{0}}(0)$ must be zero in this case, since the asymptotic expression for $F(s)$ must be zero when $s=0$. Otherwise, $F(0) \sim c_{i_{0}}(0)(1 / \bar{\gamma})^{i_{0} / \theta}[\ln (\bar{\gamma})]^{r} \neq 0, \forall \bar{\gamma}>0$, which is against our definition of outage as given in (6). Hence, the asymptotic expression for $F_{c o m b}(s)$ is

$$
\begin{aligned}
& F_{\text {comb }}(s)=\int_{0}^{s} f_{\text {comb }}(x) \mathrm{d} x \\
& \sim\left\{K^{\frac{1}{M}} c_{i_{0}}(s)\left(\frac{1}{\bar{\gamma}}\right)^{\frac{i_{0}}{\theta}}[\ln (\bar{\gamma})]^{r}\right\}^{M} .
\end{aligned}
$$

According to (17), we can employ the same method to obtain the asymptotic expression for $F_{p s}(s)$ corresponding to $f_{p s}(s)$ at high SNR. It can be easily derived that $F_{p s}(s) \sim$ $F_{\text {comb }}(s)$ and has exactly the same asymptotic expression as given in (21). As a consequence, we have proved that the asymptotic outage performances at high SNR produced by combined and per-subcarrier selections are the same, as long as their CDFs of the i.i.d. end-to-end SNR, $F(s)$, can be expanded as the form given in (18).

\section{Numerical Results and Discussion}

To verify our analysis in Section III, we employ Monte Carlo simulation methods to numerically study the outage performances of the worst subcarrier of OFDM systems employing per-subcarrier and combined bulk/per-subcarrier selection strategies respectively. Meanwhile, the asymptotic outage performance at high SNR is also taken into account in our simulations. In particular, we let $K=8, s=1$, $\mu_{1}=\mu_{2}=2, N_{0}=1$ for all simulations and $M \in\{3,5\}$ to observe the effect of $M$ on the outage performance.

In addition, all details that have been given in previous sections are under the assumption of i.i.d. channels. However, this might not be the case in practice owing to the insufficient physical separations among relays [15]. Therefore, in order to examine the robustness and practicality of the combined selection strategy, we simulated its performance over spatially equally correlated channels as well ${ }^{3}$. To model the correlation

\footnotetext{
${ }^{3}$ We maintain independence in frequency as it mimics a block fading scenario, a common model in OFDM systems that utilize a resource block structure, e.g., LTE.
}

phenomenon, we can construct the equally correlated Rayleigh fading channel by [16]

$$
\begin{aligned}
w(m, k) & =\left[\sqrt{1-\rho_{i}} x_{i}(m, k)+\sqrt{\rho_{i}} x_{i 0}(k)\right] \\
& +j\left[\sqrt{1-\rho_{i}} y_{i}(m, k)+\sqrt{\rho_{i}} y_{i 0}(k)\right]
\end{aligned}
$$

where $\left\{w(m, k), \mu_{i}\right\}=\left\{h(m, k), \mu_{1}\right\}$ or $\left\{w(m, k), \mu_{i}\right\}=$ $\left\{g(m, k), \mu_{2}\right\}$ corresponding to the first and the second hops; $i \in\{1,2\} ; j=\sqrt{-1} ; x_{i}(m, k), y_{i}(m, k) \sim \mathcal{N}\left(0, \mu_{i} / 2\right)$ are i.i.d.; $x_{i 0}(k), y_{i 0}(k) \sim \mathcal{N}\left(0, \mu_{i} / 2\right)$ are i.i.d. and serve as a bridge to correlate all channels. Hence, $\forall m \neq n$ we have $\mathbb{E}\left\{w(m, k) w^{*}(n, k)\right\} / \sqrt{\mathbb{E}\left\{|w(m, k)|^{2}\right\} \mathbb{E}\left\{|w(n, k)|^{2}\right\}}=\rho$, which is the common cross-correlation coefficient over all channels. In simulations, we let $\rho \in\{0,0.8\}$.

The simulation results corresponding to the three relay protocols are presented in Figs. 2, 3 and 4, respectively ${ }^{4}$. From these three figures, we can summarize some key points with respect to the combined selection strategy. First, for all three types of relays, it is clear that for the cases when $M=3$ and $M=5$, the outage probability of the system employing combined selection adheres to the equivalence principle over uncorrelated channels. Moreover, increasing the number of relays $M$ will yield a better outage performance, again as expected. Note, however, that an increase in $M$ does not mean that the number of utilized relays increases for the combined selection system since $|\mathcal{L}|=2$. We also note from the figures that the equivalence principle appears to hold in correlated channels as well, thus suggesting that combined selection is a robust, practical solution for a broad range of applications. Finally, the asymptotic outage probability of the VG relaying scenario shown in Fig. 4 is quite close to the performance of the DF relaying scenario as given in Fig. 2, which agrees with our analysis (cf. (12) and (16)).

\section{CONClusion}

In this paper, we considered the strategy of combined bulk/per-subcarrier relay selection for $\mathrm{DF}, \mathrm{FG} \mathrm{AF}$, and $\mathrm{VG}$ AF cooperative relay networks. Through analyzing the outage probability at high SNR, we have shown that combined relay selection satisfies the equivalence principle, i.e., it achieves the same performance at high SNR as per-subcarrier selection, but where only two out of $M$ relays are active rather than the full set. Furthermore, a generalized situation without specifying the relaying protocol is also analyzed in this paper, and we proved that the equivalence principle is generally valid as long as the CDF of the i.i.d. end-to-end SNR, $F(s)$, can be expanded as the form given in (18). Hence, it is possible to achieve very good performance at a reduced system complexity. Numerical results validated our analysis and furthermore demonstrated that the equivalence principle appears to hold in spatially correlated channels as well. Our future work will focus on the proof of the equivalence principle over spatially correlated channels.

\footnotetext{
${ }^{4}$ Here, more higher order terms for FG AF case are kept in order to illustrate the convergence between numerical and asymptotic results within a reasonable SNR range.
} 


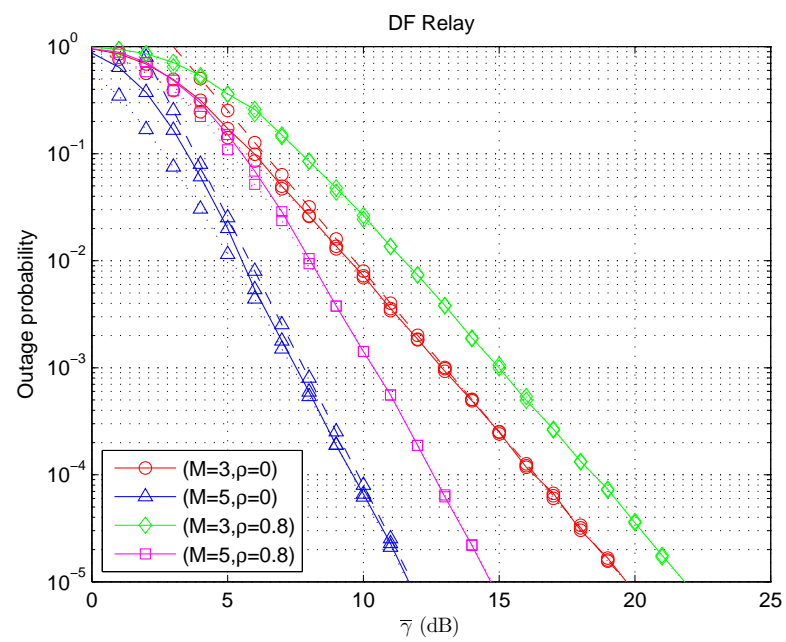

Fig. 2. DF relay case: outage probability vs. SNR for per-subcarrier and combined bulk/per-subcarrier selection systems; (a) solid line: combined selection results, (b) dotted line: per-subcarrier selection results, (c) dashed line: asymptotic results.

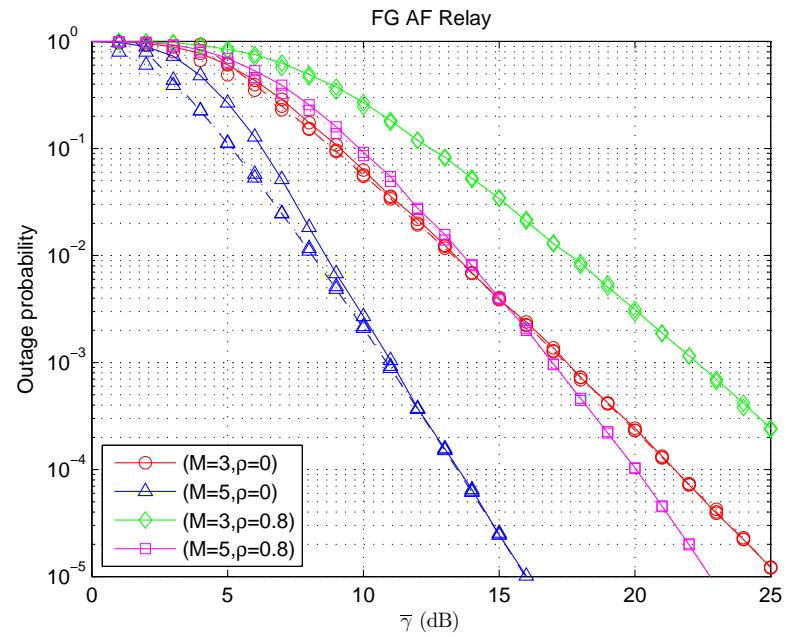

Fig. 3. FG AF relay case: outage probability vs. SNR for per-subcarrier and combined bulk/per-subcarrier selection systems; (a) solid line: combined selection results, (b) dotted line: per-subcarrier selection results, (c) dashed line: asymptotic results.

\section{ACKNOWLEDGMENT}

This work was supported by the FP7 DIWINE project (Grant Agreement CNET-ICT-318177), the grant from China Scholarship Council (No. 201508060323), Edgell Sheppee Fund and the fund from Harris Manchester College.

\section{REFERENCES}

[1] A. Chandra, C. Bose, and M. Bose, "Wireless relays for next generation broadband networks," IEEE Potentials, vol. 30, no. 2, pp. 39-43, March 2011.

[2] G. Chen, Y. Gong, and J. Chambers, "Study of relay selection in a multicell cognitive network," IEEE Wireless Communications Letters, vol. 2, no. 4, pp. 435-438, Aug. 2013

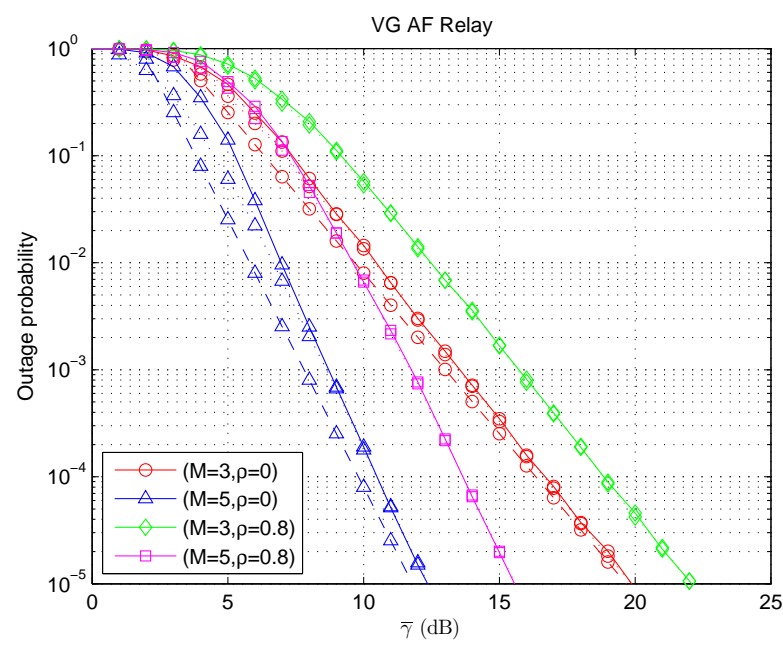

Fig. 4. VG AF relay case: outage probability vs. SNR for per-subcarrier and combined bulk/per-subcarrier selection systems; (a) solid line: combined selection results, (b) dotted line: per-subcarrier selection results, (c) dashed line: asymptotic results.

[3] G. Chen and J. Chambers, "Outage probability in distributed transmission based on best relay pair selection," IET Communications, vol. 6, no. 12, pp. 1829-1836, Aug. 2012.

[4] P. Guo, Y. Bai, Z. Ma, S. Wu, and S. Dang, "Relay technology for multicarrier systems: a research overview," in Proc. IEEE C3IT, Hooghly, India, Feb. 2015.

[5] Y. Li, W. Wang, and F.-C. Zheng, "Combined bulk and per-tone relay selection in cooperative OFDM systems," in Proc. IEEE ICCC, Beijing, China, Aug 2012.

[6] J. Coon and M. Sandell, "Combined bulk and per-tone transmit antenna selection in OFDM systems," IEEE Communications Letters, vol. 14, no. 5, pp. 426-428, May 2010.

[7] Y. Yan, C. Gan, C. Zuo, S. Wu, and S. Dang, "Analysis of uniform and optimal power allocation strategies for decode-and-forward relay considering direct transmit link," in Proc. IEEE ICEIT, Marrakech, Morocco, March 2015.

[8] W. Yang and Y. Cai, "Distributed relay selection in OFDM-based cooperative wireless networks," in Proc. IEEE WiCOM, Chengdu, China, Sept. 2010.

[9] D. Simmons, D. Halls, and J. Coon, "OFDM-based nonlinear fixed-gain amplify-and-forward relay systems: SER optimization and experimental testing," in Proc. IEEE EuCNC, Bologna, Italy, June 2014.

[10] M. O. Hasna and M.-S. Alouini, "Performance analysis of two-hop relayed transmissions over Rayleigh fading channels," in Proc. IEEE VTC Fall, vol. 4, 2002, pp. 1992-1996 vol.4.

[11] S. Dang, J. P. Coon, and D. E. Simmons, "Combined bulk and per-tone relay selection in super dense wireless networks," in Proc. IEEE ICC, London, UK, June 2015.

[12] S. Dang, D. Simmons, and J. P. Coon, "Comparison of multicarrier relay selection schemes in super dense networks," in Proc. IEEE CAMAD 2015, Guildford, United Kingdom, 2015.

[13] D. Simmons and J. Coon, "Two-way ofdm-based nonlinear amplify-andforward relay systems," IEEE Transactions on Vehicular Technology, vol. PP, no. 99, pp. 1-1, 2015.

[14] G. Huang, Y. Wang, and J. Coon, "Performance of multihop decodeand-forward and amplify-and-forward relay networks with channel estimation," in Proc. IEEE PacRim, Victoria, BC, Canada, Aug. 2011.

[15] B. Nguyen, R. Afolabi, and K. Kim, "Dependence of outage probability of cooperative systems with single relay selection on channel correlation," IEEE Communications Letters, vol. 17, no. 11, pp. 2060-2063, November 2013.

[16] Y. Chen and C. Tellambura, "Distribution functions of selection combiner output in equally correlated Rayleigh, Rician, and Nakagami$\mathrm{m}$ fading channels," IEEE Transactions on Communications, vol. 52 , no. 11, pp. 1948-1956, Nov 2004. 